\title{
Infertility treatment for HIV-positive couples: far from immaculate conception
}

\author{
Aakta Patel, Third Year Medical Student, St Bartholomew's and The Royal London School of Medicine, University of \\ London, Turner Street, London E1 2AD, UK
}

Correspondence: Aakta Patel.E-mail: ha0213@qmul.ac.uk

Journal of Family Planning and Reproductive Health Care 2003; 29(4): 239-241

\section{Introduction}

The issue of infertility diagnosis in human immunodeficiency virus (HIV)-positive couples is medically and ethically complicated. Studies show that although many in vitro fertilisation (IVF) units in the UK screen patients for HIV, only a handful are prepared to treat couples if one or other partner tests positive for the virus. ${ }^{1}$

The Human Fertilisation and Embryology Act 1990 requires the welfare of the child to be taken into account before treatment starts, but it does not exclude any category of woman from being considered for infertility treatment. In the case of HIV the primary concerns are shortened life expectancy of the infected parent(s) and the risk of viral transmission to either the uninfected partner or offspring. ${ }^{1}$ Even if the virus is not transmitted the consequences may be devastating, since the child may lose one or both parents prematurely to acquired immunodeficiency syndrome (AIDS), and the emotional and financial resources of the family are often depleted.

The ethical dilemmas these issues raise have provided sufficient grounds for most units offering assisted conception to close their doors to patients infected with HIV. ${ }^{1}$ Clearly not all patients infected with HIV will be suitable for infertility treatment, but whether couples are offered assisted conception in the UK is still very much a lottery.

\section{Time trends}

At the beginning of the AIDS epidemic most infections occurred in male patients. The picture is changing persistently and rapidly. In 2001, the World Health Organization reported 4.3 million new HIV infections in adults globally, $41 \%$ of which were in women. ${ }^{2}$ The proportion of newly diagnosed cases of HIV that are heterosexually acquired continues to rise in the UK. Figures show that in the year $2000,46 \%$ of the 2868 newly diagnosed cases were likely to have been acquired heterosexually, the majority being in people from, or people who have travelled in, high-prevalence areas, namely sub-Saharan Africa. ${ }^{3}$

AIDS continues to ravage the Third World, but medical advances available to Westerners with HIV mean they now face very different prospects. The perception of HIV infection has changed from an acute lethal infection to a chronic illness, the majority $(86 \%)$ of those individuals infected being of reproductive age (15-44 years). ${ }^{4}$ The introduction of highly active antiretroviral therapy has decreased morbidity and mortality, as have protocols for reducing the risk of mother-to-child transmission. In view of these advances some HIV-infected individuals are considering reproduction, so fertility issues become inevitable. ${ }^{3}$

\section{Treatment advances}

In 1981, when AIDS was first reported, there was no effective therapy for patients infected with HIV. Mother-to- child transmission of HIV may occur in utero, intrapartum or by breastfeeding in $15-40 \%$ of infected pregnant women. Factors associated with an increased risk of perinatal HIV transmission include advanced maternal disease status, infant exposure to maternal blood, prolonged duration of ruptured membranes, and increased maternal viral load at delivery. ${ }^{2,5}$ It became apparent that approximately $25 \%$ of women with HIV infection who gave birth transmitted the virus to their children. ${ }^{2}$

Since then the prognosis for both infected mothers and their offspring has improved substantially. Aggressive treatment with highly active antiretroviral drugs has seen a decline in the AIDS mortality widely portrayed in both the lay press and medical literature. In 1994, the AIDS Clinical Trials Group Study was closed by the study's data and safety monitoring board when the efficacy of zidovudine $(\mathrm{AZT})$ - a nucleoside reverse transcriptase inhibitor, intended for prevention of mother-to-child transmission was found to be even more dramatic than anticipated. ${ }^{6}$

Connor et al. ${ }^{6}$ showed that the drug dramatically reduced vertical transmission from $16-24 \%$ to $5-8 \%$ when given to HIV-positive pregnant women during the second and third trimesters, and to their newborns for 6 weeks. Subsequent studies have confirmed and extended these results. It is likely that zidovudine may exert its protective effect by reducing maternal HIV-1 levels prior to delivery, inhibiting HIV-1 blood and secretions in the fetus during labour and delivery, and preventing the virus from establishing infection in the fetus and the infant. ${ }^{4}$

Despite the clear benefits of antiretroviral therapy, the development of drug resistance is common. The regimens are also burdensome and have serious side effects, hence not all infected individuals will benefit. ${ }^{3,4}$

A recent meta-analysis of studies conducted in North America and Europe concluded that elective Caesarean section combined with antiretroviral treatment can decrease the vertical transmission rate to $2 \%$, compared with $7.6 \%$ in children of treated women who deliver vaginally. Subsequent studies have found that Caesarean section is not needed to lower the risk of transmission if viral levels in the pregnant woman are undetectable. ${ }^{4}$

\section{Access to services}

In general, infertility clinics are still biased against patients infected with HIV. ${ }^{7}$ Clinics providing assisted conception as of December 2000 were identified from the records of the Human Fertilisation and Embryology Authority, which licenses the use of such techniques in Britain. Of the 75 clinics sent a questionnaire, responses were received from 57 $(76 \%)$. Of these, 41 units $(72 \%)$ had a policy on treating patients infected with HIV. Most (61\%) of these units had not seen a patient infected with HIV in the previous year, but of those who had only $44 \%$ agreed to treat couples where only the man was infected with HIV (Table 1). ${ }^{1,7}$ The consensus is still to encourage donor insemination rather than offering assisted conception techniques to such couples. 
Table 1 Responses from infertility clinics asked whether they would offer infertility investigations and treatment to couples when one or both partners were infected with $\mathrm{HIV}^{7}$ (Table reprinted, with permission, from the British Medical Journal)

\begin{tabular}{llll}
\hline Question & \multicolumn{3}{l}{ Response (\%) } \\
\cline { 2 - 5 } & Yes & No & None \\
\hline Would you offer infertility investigations & & & \\
to a couple when: & & & \\
$\quad$ The male partner is HIV-positive? & $33(58)^{\mathrm{a}}$ & $17(30)$ & $7(12)$ \\
$\quad$ The female partner is HIV-positive? & $25(44)$ & $24(42)$ & $8(14)$ \\
$\quad$ Both partners are HIV-positive? & $24(42)$ & $25(44)$ & $8(14)$ \\
Would you offer infertility treatment & & & \\
to a couple when: & & & \\
$\quad$ The male partner is HIV-positive? & $25(44)^{\mathrm{a}}$ & $26(46)$ & $6(10)$ \\
$\quad$ The female partner is HIV-positive? & $14(25)$ & $36(63)$ & $7(12)$ \\
Both partners are HIV-positive? & $12(21)$ & $38(67)$ & $7(12)$ \\
\hline
\end{tabular}

$\mathrm{a} p=0.001$.

\section{Ethical dilemmas}

Currently no established guidelines exist for defining access to fertility care for individuals infected with HIV. What, then, should be the guidelines for deciding the appropriateness of assisted reproduction for couples with HIV infection? If one considers the two most frequently cited barriers to the use of assisted reproduction technology - poor fetal prognosis and poor maternal prognosis - it becomes evident that the refusal to offer services to couples with HIV infection may be unjust. ${ }^{5}$

\section{Maternal prognosis}

With regard to the prognosis of women with HIV infection, there are reasonable arguments for the treatment of infertility. With access to current therapies, it is clear that life expectancy is increasing for many couples with HIV infection. Although the precise number of years a given person will survive cannot be known for sure, current estimates suggest that a disease previously associated with certain death is compatible with a life expectancy of at least 20 years from time of diagnosis. ${ }^{1,7}$

There are many similarities between HIV and other oncefatal diseases afflicting women in their reproductive years such as diabetes mellitus, cystic fibrosis, congenital heart disease and breast cancer. Cardiac disease and cystic fibrosis, in particular, may worsen considerably during pregnancy, with effects on both maternal and fetal health. ${ }^{1}$ Yet fertility treatment is rarely refused in these cases; the issue is the degree to which a potential parent's long-term health has to be guaranteed before assisted reproduction becomes reasonable. Should a physician offer assisted reproduction techniques to a 45-year-old woman with advanced diabetes? If the answer is yes, then it is reasonable to suggest that a well-treated woman with HIV infection, whose prognosis is not completely dissimilar, should also be treated.

\section{Fetal prognosis}

The major question regarding fetal prognosis is at what point do fetal and neonatal risks become unacceptably high that infertility treatment should not be offered? Regarding the possibility of transmission of a potentially lethal illness from mother to child, it is unlikely for example, that a couple who were both carriers of the gene for Tay-Sachs disease (or other similar autosomal-recessive traits) would be denied access to assisted reproduction techniques merely on the basis of the risk to the fetus. The $25 \%$ risk of the fetus having a lethal disease far exceeds the current risk of $2 \%$ among the fetus's of HIV-infected mothers who are appropriately treated during pregnancy. ${ }^{4,6}$ So, one may ask, what is all the fuss about? It seems the argument is more complex than simply a matter of comparative statistics.

\section{Couples in need}

Two broad groups of patients infected with HIV are likely to request infertility treatment. The first group consists of couples in which only one partner is infected with HIV (sero-discordant couples) who would like to have children but who wish to avoid transmission of the virus to the uninfected partner; they may not be subfertile. ${ }^{7}$

Where there is HIV discordance the couple should be made aware of the inherent risk of horizontal transmission of HIV. The 'per act' risk of sexual transmission is difficult to quantify; a constellation of factors including stage of HIV infection, response to any prescribed antiretroviral therapy, type of sex, and the presence of other local infection) are often involved. Concurrent sexually transmitted infection in either or both the HIV-infected and HIV-uninfected partners is strongly associated with an increased risk of transmission. ${ }^{3}$

The second group consists of sero-discordant couples that are subfertile and have tried to conceive spontaneously (with the attendant risks) without success, and subfertile couples in which both partners are infected with HIV who would like to have children. ${ }^{7}$

\section{Sero-discordant males}

The female partner of an HIV-positive man runs a $0.1-0.2 \%$ risk of acquiring HIV in an act of unprotected intercourse; this excludes the presence of risk factors which increase transmission rate, for example, concomitant genital infection. ${ }^{1}$ If a man is HIV-positive and his female partner is HIV-negative, the risk of transmitting the virus to the female partner appears to be reduced but not eliminated by using condoms during sexual activity, except during ovulation. Even though some HIV-discordant couples have established pregnancies through timed unprotected intercourse without infecting the negative partner or child, this practice is unsafe and not recommended. 4

Until now, sperm donation remains the first choice of fertility treatment for men who are HIV-positive. Although the issue is controversial, there is little evidence to support HIV being able to attach to, or infect, spermatozoa. In men infected with HIV, virus is present in semen as free virus in the seminal plasma and as cell-associated virus in the non-sperm cells. Recent reports have described specific methods for sperm preparation and testing that can substantially reduce the chance of HIV transmission to the female partner and child. 2,3

In 1998, scientists in Milan were the first to use a density gradient and swim-up technique to obtain sperm, which were then tested by polymerase chain reaction assays for the presence of HIV. Semprini et al. ${ }^{8}$ noted that a highly significant reduction in the risk of viral transmission is achieved if spermatozoa are first washed free of seminal plasma and non-sperm cells before insemination into the woman at the time of ovulation..$^{5,8}$

As a risk-reduction option the results are convincing. Three hundred healthy children have now been born after more than 3000 cycles of sperm washing and intrauterine insemination treatment or IVF, with no reported seroconversions in either partner or children. ${ }^{1}$ Marina et al. reported similar results in 63 women using a similar method of sperm processing and this technique of 'sperm washing' is now practised in several centres in Europe, including the Chelsea and Westminster unit in London, UK. ${ }^{1,8}$

More data are needed to demonstrate the complete efficacy of these sperm preparation techniques. Until then, couples must still be cautioned about the potential risk of HIV transmission to the uninfected partner and to their offspring. Couples seeking the safest methods to prevent transmission of the virus when the male partner is HIVpositive should be counselled about using donor sperm, considering adoption, or even against having children. 5,7 


\section{Sero-discordant females}

If a woman is HIV-positive and her male partner is HIVnegative, transmission of infection to the male partner can be avoided by using self-insemination with the partner's sperm, during the fertile period of the cycle. Some couples prefer to 'medicalise' the process as little as possible and choose to extract semen from spermicide-free condoms for insemination after intercourse. ${ }^{3}$

Irrespective of such measures, the resulting pregnancy may still pose some risk to the HIV-positive woman and her child, because opportunistic infections occurring during pregnancy can be devastating to the woman and fetus. 4 Potential teratogenic effects of antiretroviral drugs taken during pregnancy remain an issue - serious adverse effects appear rare, although mitochondrial cytopathy leading to neonatal death has been documented. Amniocentesis, a procedure commonly recommended to women older than 35 years of age, also carries the risk of viral transmission to the fetus since the needle is passed through the HIV-positive woman's abdominal cavity into the amniotic sac. ${ }^{1,4}$

When both partners are infected with HIV, the risk of the virus being transmitted to the child can be reduced by the methods described previously - by the mother taking antiretroviral drugs throughout pregnancy and labour, having a Caesarean section, by the child receiving antiretroviral therapy at birth, and by avoidance of breastfeeding. ${ }^{7}$

\section{HIV testing for gamete donors}

It is important to mention that testing for HIV and other sexually communicable diseases is ethically justified for donors, to protect the health of the gamete recipients. Testing donors and recipients for potentially transmittable infectious conditions can be reassuring to all parties involved in assisted reproductive technology and should be strongly encouraged. It is especially important to test persons who are considered at high risk for HIV infection, such as those who have a history of repeated sexually transmitted diseases, multiple sexual partners without barrier protection, bisexual behaviour or intravenous drug use. ${ }^{2,8}$

\section{HIV testing for parents}

It is ethically appropriate for practitioners to encourage HIV testing for all couples who want to have children, not just those who request infertility treatment. To mandate that people be tested solely because they request medical assistance in having a child would infringe on their personal liberty, and introduce a dubious distinction between those who seek treatment for infertility and those who do not. ${ }^{4}$ Since there are means to significantly lessen the chance of HIV transmission to an uninfected partner and to offspring, a strong argument exists that all couples should consider HIV testing as part of responsible parenting. ${ }^{2}$

Often associated with testing is the presumed stigma of some past sexual or drug-related misbehaviour. Clinicians have a responsibility to educate their patients about the possible means by which infections can be acquired, and the advantages of knowing the test results before a pregnancy is established. ${ }^{9}$

\section{Conclusions}

HIV infection is classified as a chronic disease. It is treatable but not yet curable. Significant advances in HIV treatment appear to have delayed the onset of AIDS and its consequences in many, but not all, infected persons. ${ }^{4}$ This has led to increasing demand for couples - in which one or both partners are infected - to have access to the same fertility advice and treatment as non-infected individuals, thus enabling them to conceive with the minimum of risk to their partners or children. ${ }^{2}$

As with any couple presenting for evaluation and treatment, both persons may have normal fertility potential or one or both may have impaired fertility. If an HIV-positive couple requests medical advice regarding pregnancy, they must be informed about the risks to the pregnant woman and the risk that a child could become infected. If the viral load can be suppressed to undetectable levels in both partners then the couple may have a child who is free of HIV. ${ }^{10}$

Although reproductive assistance may play a role in preventing viral transmission to offspring, such techniques cannot eliminate the risk entirely. Those who assess the ethics of assisting such patients to have children must address the question of whether offspring born with HIV are harmed despite the preventive steps taken. ${ }^{4}$ Until sperm preparation techniques prove completely effective there may be no way, short of refraining from reproduction altogether, to completely prevent some cases of HIV transmission. ${ }^{8}$

When an affected couple requests assistance to have their own genetically related child they are best advised to seek care at institutions with facilities that can provide the most effective evaluation, treatment and follow-up. It is currently recommended that all infertile couples should be tested for HIV, not for the purpose of excluding HIVpositive patients from treatment, but to offer them preconceptional counselling, prior to accepting riskreducing fertility treatments and antenatal care. ${ }^{1,10,11}$ This can be best achieved through a multidisciplinary approach including an obstetrician, fertility specialist, AIDS specialist, paediatrician and counsellor. Together the team can sufficiently evaluate the couple's motivation for having a child, and inform them about all the risks associated with their intended method of conception. Alternatively, the couple may be advised to consider other options such as donor sperm, adoption or not having children. ${ }^{4}$

\section{The future?}

With the introduction of protease inhibitors for the treatment of AIDS, the scene is set for their use in zidovudine-resistant patients with a high viral load. Combination chemotherapy will have a major impact on HIV transmission, decreasing maternal viral load and perhaps reducing fetal transmission. ${ }^{7}$ Long-term studies highlighting the impact of potential HIV transmission on sero-discordant couples seeking reproduction are also required, if advances are to be made in this exciting but controversial area of HIV medicine.

\section{References}

1 Gilling-Smith C, Smith R, Semprini A. HIV and infertility: time to treat. $B M J$ 2001; 322: 566.

2 Rizk B, Reeves Dill S. Infertility among HIV-positive women: counselling HIV patients pursuing infertility investigation and treatment. Hum Reprod 1997; 12: 415-416.

3 Moore A, Madge S, Johnson M. HIV and pregnancy. The Obstetrician \& Gynaecologist 2002; 4: 197-200.

4 Ethics Committee of the American Society for Reproductive Medicine. Human immunodeficiency virus and infertility treatment. Fertil Steril 2002; 77: 2.

5 Minkoff H, Santoro N. Ethical considerations in the treatment of infertility in women with human immunodeficiency virus infection. $N$ Engl J Med 2000; 342: 1748-1750.

6 Connor E, Sperling R, Gelber R, et al. for the Paediatric AIDS Clinical Trials Group Protocol 076 Study Group. Reduction of maternal-infant transmission of human immunodeficiency virus type 1 with zidovudine treatment. N Engl J Med 1994; 331: 1173-1180.

7 Apoola A, Tenhof J, Allan PS. Access to infertility investigations and treatment in couples infected with HIV: questionnaire survey. $B M J$ 2001; 323: 1285.

8 Semprini AE. Insemination of HIV-negative women with processed semen of HIV-positive partners. Lancet 1993; 341: 1343-1344.

9 Mandelbrot L, Heard I, Henrion-Geant E, et al. Natural conception in HIV-negative women with HIV-infected partners. Lancet 1997; 349: 850-851.

10 Hogg RS, Heath KV, Yip B, et al. Improved survival among HIVinfected individuals following initiation of antiretroviral therapy. JAMA 1998; 279: 450-454.

11 Palella FJ, Delaney KM, Moorman AC, et al. Declining morbidity and mortality among patients with advanced human immunodeficiency virus infection. N Engl J Med 1998; 338: 853-860. 